\title{
Paraneoplastic cerebellar degeneration. \\ I. A clinical analysis of 55 anti-Yo antibody-positive patients
}

\author{
K. Peterson, MD; M.K. Rosenblum, MD; H. Kotanides, MS; and J.B. Posner, MD
}

\begin{abstract}
Article abstract-We reviewed the clinical findings in 55 patients with cerebellar degeneration associated with the anti-Yo antibody (an anti-Purkinje cell antibody identified in this study by histochemistry and Western blot). The patients were all women, 26 to 85 years old. Fifty-two of them proved to have malignancies, almost exclusively breast or gynecologic cancers and usually confined to the involved organs and local lymph nodes. One woman had adenocarcinoma of the lung, and in three no malignancy has yet been identified. In 34 of 52 patients with cancer, the neurologic syndrome preceded the diagnosis of cancer and in many led to that diagnosis. Patients subacutely developed a pancerebellar disorder that was substantially disabling in most, with 37 of 48 assessable patients being unable to walk or sit unassisted. Laboratory evaluation revealed lymphocytic pleocytosis in 35 patients, with eventual cerebellar atrophy on imaging studies in seventeen. The disabling neurologic syndrome generally did not respond to treatment, but the cancer was often successfully treated. The presence of the anti-Yo antibody in patients with cerebellar symptoms warrants an aggressive approach to diagnosis and treatment of the underlying cancer, as many are curable at the time neurologic symptoms develop.
\end{abstract}

NEUROLOGY 1992; 42:1931-1937

Paraneoplastic cerebellar degeneration (PCD) is a clinical syndrome in which cerebellar dysfunction is associated with identifiable or occult cancer but without direct involvement of the nervous system by the cancer. Typically, the disorder evolves subacutely, causes severe pancerebellar dysfunction, and is associated with extensive Purkinje cell loss, sometimes accompanied by meningeal and deep cerebellar nuclear inflammatory infiltrates..$^{1-3} \mathrm{Up}$ until 1982, Henson and Urich ${ }^{3}$ were able to identify only 50 pathologically proven cases. Even with recent renewed interest in the disorder, the number of patients reported in the literature is about two hundred. ${ }^{4}$ Although the disorder is rare, Henson and Urich $^{3}$ estimate that at least 50\% of middle-aged patients who present with a subacutely developing pancerebellar disorder prove within a few years to have cancer.

It is increasingly apparent that PCD, despite its rarity, subdivides into several disorders that can be distinguished clinically and immunologically, and that differ in their prognoses and in the types of associated malignancies. The clinical findings in $\mathbf{5 5}$ patients with one of these disorders, PCD associated with a particular autoantibody designated "antiYo," are described in this paper. The two papers that follow (pages 1938 and 1944) describe PCD associated with Hodgkin's disease, and an unusual variant of PCD associated with small-cell lung cancer (SCLC) in which the majority of patients also have the Lambert-Eaton myasthenic syndrome. A fourth disorder, in which PCD may be the presenting symptom of a more widespread encephalomyelitis associated with SCLC and an antinuclear antibody called "anti-Hu," is detailed elsewhere..$^{5,6}$ Recognizing the specific variant of PCD on clinical grounds and with immunologic testing often allows the physician to better predict the clinical course and direct the search for an underlying occult malignancy.

Methods. Patients. The medical records of all antiYo-positive patients (defined by immunohistochemistry and immunoblotting) identified in our laboratory were reviewed. Eighteen of these patients had been reported previously. ${ }^{7}$ Since only 10 of the 55 patients were exam-

From the Departments of Neurology (Drs. Peterson and Posner) and Pathology (Dr. Rosenblum) and the George C. Cotzias Laboratory of NeuroOncology (H. Kotanides and Dr. Posner), Memorial Sloan-Kettering Cancer Center, and the Department of Neurology and Neuroscience (Drs. Peterson and Posner), Cornell University Medical Coilege, New York, NY.

Supported in part by NIH Grant \#NS26064.

Presented in part at the 43rd annual meeting of the American Academy of Neurology, Boston, MA, April 1991.

Received January 17, 1992. Accepted for publication in final form March 24, 1992.

Address correspondence and reprint requests to Dr. Jerome B. Posner, Department of Neurology, Memorial Sloan-Kettering Cancer Center, 1275 York Avenue, New York, NY 10021. Dr. Peterson's current address is Department of Neurology, University of Minnesota Medical School, Minneapolis, MN. 
ined by one or more of the authors, referring physicians sending sera and/or CSF for antibody testing provided the neurologic and oncologic profiles. Telephone calls to the physicians most recently earing for the patients provided follow-up as of December 1991. Microscopic slides and blocks of the cerebellum obtained at autopsy in three patients and tumor tissue in seven patients were studied directly by us.

Serum and CSF database. Approximately 2,000 sera and/or CSF samples have been examined in our laboratory for the presence of anticerebellar antibodies. These samples were sent from throughout the United States and many other countries by physicians suspecting the diagnosis of a neurologic paraneoplastic syndrome. Some patients had known cancers whereas others did not. All samples were studied by immunofluorescence or immunoperoxidase histochemistry, and by Western blotting against Purkinje cell extracts. A seareh of the databank also identified all patients in 1989 and 1990 with cerebellar syndromes with or without cancer, and all patients with ovarian or breast cancer with or without cerebellar syndromes.

Immunologic techniques. Immunohistochemistry and Western blotting techniques against Purkinje cell extracts, as well as the CDR 62 fusion protein that corresponds to the $62 \mathrm{kd}$ antigen to which the anti-Yo antibody binds, have been described extensively in previous publications from this laboratory. ${ }^{7-14}$

Results. Immunologic findings. Serum or CSF from 55 patients was positive for the anti-Yo antibody as determined by immunohistochemistry and Western blotting. In 41 of 41 patients tested, sera also reacted positively against the Yo fusion protein. ${ }^{14}$

Several patients who had atypical histochemical staining and/or atypical immunoblotting were excluded. Excluded patients included one whose serum was positive by histochemistry but reacted on Western blotting against Purkinje cell extracts with a band of $68 \mathrm{kd}$ (but not $34 \mathrm{kd}$ ), and was negative against the Yo fusion protein. Three patients had atypical Purkinje cell cytoplasmic staining and were negative on immunoblotting. Two patients with PCD showed neuronal nuclear staining and a band at 35 to $40 \mathrm{kd}$ on Western blot (typical of the anti-Hu antibody) but were negative when tested against the Yo fusion protein, and one other with atypical nuclear staining was negative with both immunoblotting techniques.

Thirteen patients with Hodgkin's disease and PCD, 45 patients with breast cancer and 24 with ovarian cancer with or without cerebellar syndromes, 325 patients with cerebellar syndromes with or without cancer, and 50 normal controls (blood bank donors) were also negative by both histochemistry and immunoblotting.

Clinical results. The 55 patients with the typical anti-Yo antibody presented a stereotypic clinical syndrome. All patients were women, 26 to 85 years old.

Tumors. Fifty-two of the patients currently have histologically proven malignancies. The most common tumor, ovarian, was found in 26 of 55 patients;
13 had breast cancer, and seven had other gynecologic malignancies (four endometrial, two fallopian tube, and one mesovarium). One patient had both breast and fallopian tube cancers. One patient had an adenocarcinoma of the lung, and is without known gynecologic malignancy.

There were six patients with adenocarcinoma of unknown primary. Three of these presented with axillary nodes, likely representing breast cancer, although no primary was found in the breast. One patient with adenocarcinoma of unknown primary had had resection 2 years previously of an ovarian mass that had shown benign pathology; at autopsy, this patient was found to have had a periaortic node pathologically suggestive of ovarian cancer and had staining for CA 125 antigen, which is associated with ovarian cancers. One patient presented with carcinoma in an inguinal lymph node. One other patient had a poorly differentiated adenocarcinoma in an abdominal lymph node compatible with ovarian cancer, but no ovarian tumor was found. At autopsy 3 years later, she was found to have a hepatoma but no adenocarcinoma; the original primary was not found.

Three patients had no tumor identified. One of these patients had resection of an ovarian mass with benign histology (ovarian cystadenoma); she died 24 months after the onset of her neurologic syndrome and no cancer was found at autopsy. Two patients are still living, up to 24 months after the onset of their neurologic disorder, and have so far shown no evidence of malignancy despite serial physical and radiologic examinations.

In 34 of 52 patients with known cancer, the neurologic syndrome preceded the diagnosis of malignancy by up to 15 months, and in many of the cases prompted a search for the underlying cancer. Of these 34 patients, 16 had ovarian cancer; notably, four had microscopic disease not found by routine diagnostic testing and detected only at laparotomy. However, one patient with a negative mammogram and normal pelvic examination underwent laparoscopy with ovarian biopsy, and then hysterectomy and bilateral salpingo-oophorectomy without a malignancy being found. Four months after the initial normal mammogram, a breast abnormality was detected and biopsy revealed an intraductal carcinoma.

Most patients with known cancer had limited oncologic disease at the time of onset of neurologic symptoms, with only 7 of 52 having evidence of widely metastatic cancer. Ten patients had local disease, 26 had regional metastases, and six patients were in remission. Three patients presented with neurologic symptoms coincident with recurrence of a malignancy that had previously been in remission.

Neurologic findings. Patients presented with a characteristic pancerebellar disorder with truncal and limb ataxia, dysarthria, and nystagmus, of subacute onset ( 1 day to 16 weeks) and subsequent stabilization. Complete details of the neurologic 
examination were available to us in 48 patients. The ataxia was initially asymmetric in 20 , although in most, ataxia became symmetric as the disease progressed. Most patients were severely disabled by the neurologic syndrome, with 37 of 48 patients being unable to walk or sit unassisted due to severe ataxia; 9 of 48 could walk with some assistance, and 2 of 48 could walk unassisted but were unsteady.

All patients had some degree of horizontal nystagmus, with a rotatory or vertical component in twenty-seven. Seventeen patients were specifically reported to have downbeating nystagmus. All of the 10 patients we examined had downbeating nystagmus; we suspect that this finding was underreported in records we reviewed. Five patients reported oscillopsia and 19 patients experienced diplopia. However, limitation of extraocular movements with unilateral or bilateral abducens palsies was detected in only six. In 20 patients there was other bulbar involvement, including dysphagia in 16 and facial weakness in seven. Opsoclonus was reported in two patients, and sleep myoclonus in one other.

Signs or symptoms of involvement at other levels of the neuraxis were common but usually mild. Although accurate mental status testing was difficult to confirm because of dysarthria and motor dysfunction, 10 patients were believed clinically to have some cognitive impairment, usually emotional lability and memory deficit. Twenty-eight had extensor plantar responses or other long-tract signs. Two patients had extrapyramidal rigidity and tremor or dyskinesias. Twenty-six had hyporeflexia or mild distal sensory complaints suggesting peripheral neuropathy. Two patients had progressive visual loss. This finding was believed clinically to be a result of either optic neuropathy or retinal degeneration, although this was unconfirmed by electrographic studies.

Headache was an uncommon complaint, prominent in only six patients. Twenty patients complained of vertigo. Nausea and vomiting were debilitating symptoms in two.

None of the patients was known to have other immunologic, rheumatic, or collagen vascular diseases, save for hypothyroidism in two patients and adult-onset diabetes mellitus in one.

Laboratory evaluation. CSF examination was performed in all patients. Of the 42 patients in whom detailed CSF results were available, only seven had normal CSF. Thirty-five patients had inflammatory changes that included a predominantly lymphocytic pleocytosis (between 6 and 93 $\mathrm{WBC} / \mathrm{mm}^{3}$ ) in 26 of 42 and elevated protein (between 48 and $106 \mathrm{mg} / \mathrm{dl}$ ) in 22 of 42 patients. The ratio of CSF to serum IgG was increased in 12 of 14 patients for whom this information was available; oligoclonal bands were present in 6 of 7 patients tested, and elevated myelin basic protein reported in one.

CT and/or MRI was performed in all patients.
Table 1. Clinical findings in 55 anti-Yo-positive PCD patients

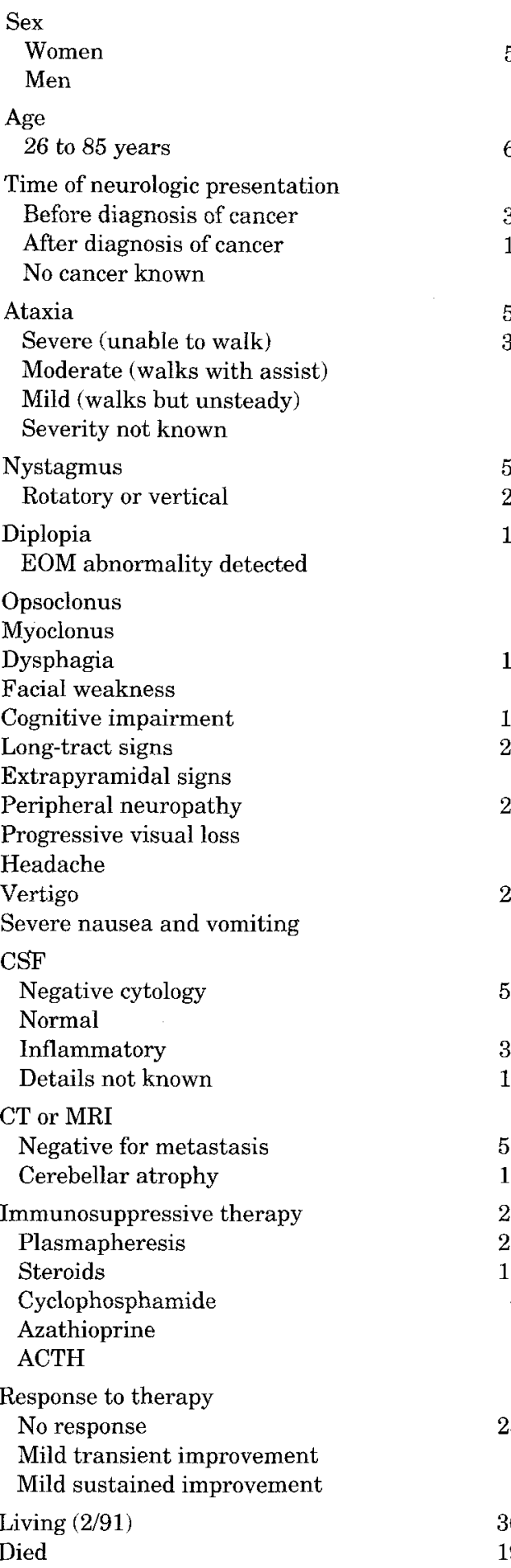

EOM Extraocular movement

Most of these studies were initially normal, although 17 patients had evidence of cerebellar atrophy on CT or MRI, particularly later in the disease process. In seven patients studied with ${ }^{18} \mathrm{~F}$-fluorodeoxyglucose positron emission tomography, the mean regional metabolic rate for glucose was sig- 


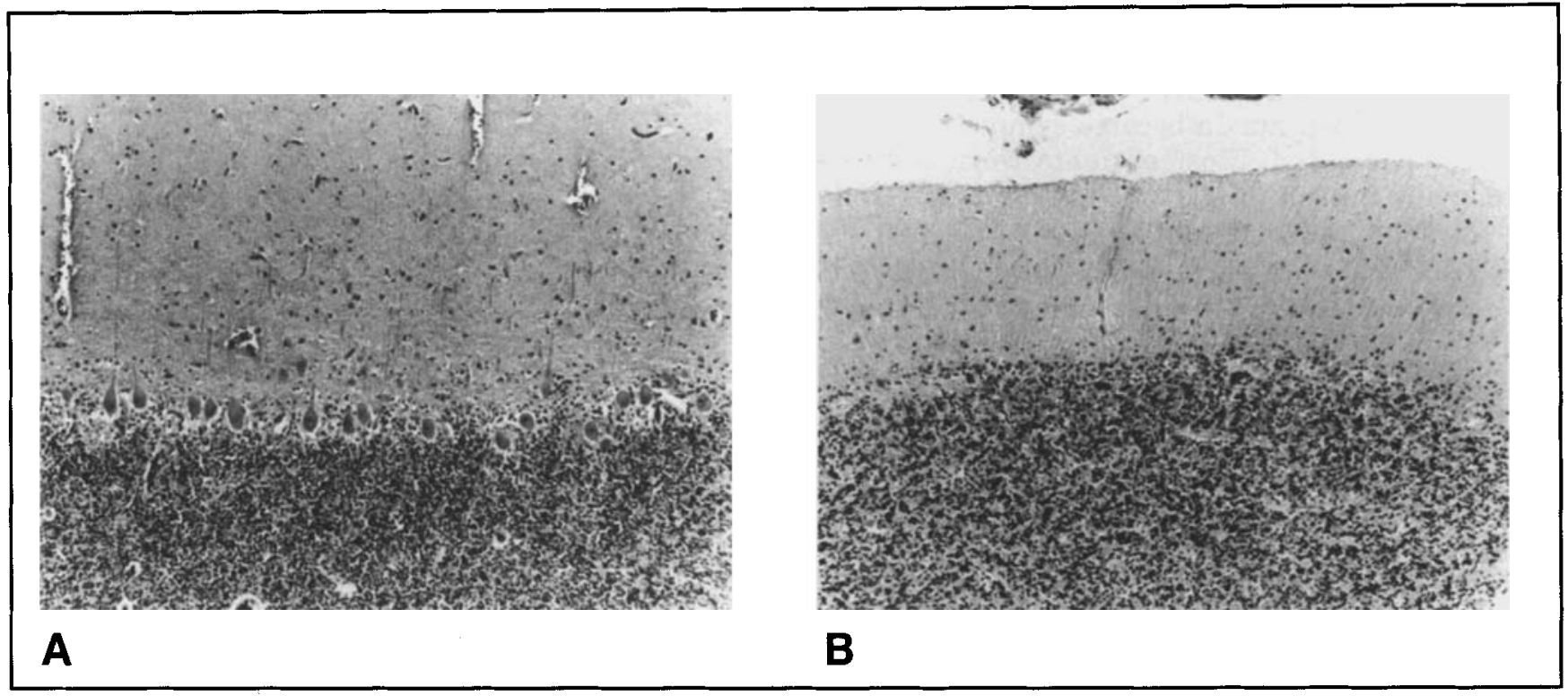

Figure. (A) Photomicrograph of normal cerebellar cortex from a 55-year-old man who died of disseminated carcinoma without neurologic involvement. Purkinje cells are prominent at the junction of the molecular and granular layers (hematoxylin-eosin; $\times 100$ before $29 \%$ reduction). (B) View of the cerebellar cortex from a patient who died with anti$Y_{O}$-positive PCD demonstrating total Purkinje cell loss. Note the absence of inflammatory infiltrates (hematoxylin-eosin; $\times 100$ before $29 \%$ reduction).

nificantly reduced in the cerebellum and several cortical regions as well, when compared with that of normal individuals. ${ }^{15}$

Treatment. On the rationale that this neurologic syndrome was autoimmune, several immunosuppressive therapies were tried in various combinations in a total of 29 patients. These included plasmapheresis in 22 patients (at least five exchanges). There was mild but transient improvement in the symptoms of ataxia and dysarthria in four patients. Another patient had a moderate sustained benefit, regaining her ability to walk with assistance. This correlated with a decrease in her serum antibody titers from 1:4,000 pre-treatment to 1:200 post-treatment. However, five other patients with documented decline in serum antibody titers after treatment showed no clinical response, possibly related to the finding that there was no corresponding decline in CSF antibody titers. ${ }^{16}$ High-dose steroids (40 to $100 \mathrm{mg} / \mathrm{d}$ for at least 2 weeks) gave mild but transient improvement in one of 17 patients treated. There was no beneficial effect of cyclophosphamide in four patients, azathioprine in two, or ACTH in one patient. One patient was reported to have had moderate improvement with treatment of the underlying tumor.

At the time of most recent follow-up, 36 patients are still living, up to 77 months after the onset of neurologic symptoms. Most of these are in remission from their malignancies but remain severely disabled neurologically. Nineteen patients have died, between 2 and 36 months after the onset of their neurologic symptoms, due to progression of their oncologic disease and inanition (table 1).

Pathology. The neuropathologic findings at autopsy were available for review in three cases and conformed to previous descriptions of PCD in patients with breast and gynecologic cancer. ${ }^{3}$ All were characterized chiefly by cerebellar cortical atrophy with near-total depletion of Purkinje cells and proliferation of Bergmann astrocytes (figure). Thinning of the granule cell population was an additional feature of two cases. Scattered small lymphocytes, mainly T-cells by immunotyping, were noted in the cerebellar leptomeninges, but in no case were inflammatory infiltrates identified in the cerebellar cortex proper. Small numbers of mature lymphocytes, again mainly T-cells, also cuffed blood vessels in the dentate nucleus but were not associated with appreciable neuronal loss. As expected, the dentate amiculum (composed normally of Purkinje cell axons) exhibited axonal depopulation and secondary demyelination in all cases.

Microscopic sections of the PCD-associated neoplasm at its primary site were available for review in seven cases (one fallopian tube, one endometrial, two mammary, and three ovarian). Both breast primaries were high-grade, poorly differentiated infiltrating adenocarcinomas of the duct type. Similarly, the endometrial, ovarian, and tubal neoplasms were found to be high-grade müllerian adenocarcinomas composed of solid and papillary elements. An unusual and striking feature common to the stroma of all seven neoplasms was a conspicuous lymphoid infiltrate dominated by mature plasma cells. As previously described, ${ }^{12}$ expression of the Yo antigen by PCD-associated neoplasms was demonstrable in immunohistochemical and Western blot assay in all 10 instances in which fresh tumor tissue was available for analysis. 


\section{Author}

Trotter et al ${ }^{20}$

1976

Stefansson et al ${ }^{19}$

1981

Brown et al ${ }^{22}$

1985

Kornguth et $\mathrm{al}^{23}$

1985

Rodriguez et $\mathrm{al}^{24}$

1986

Tanaka et al ${ }^{25}$

1986

Greenlee and Brashear ${ }^{26}$ 1983

Greenlee et al ${ }^{27} 1986$;

Greenlee and Lipton ${ }^{28}$

1986

Jaeckle et $\mathrm{al}^{8}$

1985

Cunningham et $\mathrm{al}^{9}$

1986

Bourdette and Nilaver ${ }^{29}$ 1987

Royal et al ${ }^{30}$

1987

Anderson et $\mathrm{al}^{31}$

1988

Anderson et al $^{7}$

1988

Tsukamoto et $\mathrm{al}^{32}$

1989
Tumor (no.)

Hodgkin's (1)

Hodgkin's (1)

Lung (small cell) (1)

Ovary (1)

"Gynecologic cancer" (4)

Non-Hodgkin's (1)

Ovary, breast (9)

Lung (small cell) (3)

Breast (1)

Ovary, breast (7)

Lung (small cell) (5)

Hodgkin's (5)

Benign monoclonal gammopathy

Breast (1)

Lung (adeno) (1)

Ovary (9),

breast (6),

adenocarcinoma

unknown $1^{\circ}(3)$

Lung (adeno) (1),

colon (1)

Lung (small cell) (1)

Breast (1)

Ovary (2),

uterus (2)

Breast (1),

lung (1)
Antigen distribution, titer (immunohistochemistry)

Purkinje cell cytoplasm,

1:20

Axons, dendrites in cerebral, cerebellar cortex

Purkinje cell cytoplasm; neurons in deep cerebellar nuclei, brainstem, forebrain

Purkinje cell cytoplasm

Purkinje cell cytoplasm

Purkinje cell cytoplasm

Purkinje cell cytoplasm

1:640

Purkinje and granule cell cytoplasm Purkinje and granule cell nuclei,

CNS neurons, 1:320

Purkinje cell cytoplasm, 1:1,000

Absent

Absent

Purkinje cell cytoplasm

Purkinje cell cytoplasm

Purkinje cell cytoplasm

fine granular pattern

human, rat, rabbit, bovine, $1: 3,000$

Weak staining cortical, hippocampal brainstem neurons, 1:50

Purkinje cell cytoplasm

Purkinje cell cytoplasm

All neuronal nuclei

All neuronal nuclei

Purkinje, stellate, basket cells

deep cerebellar nuclei

cortical, hippocampal neurons,

$1: 10,000$

All neuronal cells,

1:100,000
$34-38$

62-64

Human Purkinje cell

$34-38$

$62-64$

$35-40$ (anti-Hu)

$53-61$

79-84

52,58

munoblots

Mouse cerebellum, brain, pinal cord

(cerebrum)

$34-38$

$62-64$

$56,64,68,80$

30,46

38,40 
Discussion. This report confirms and extends findings previously reported on 18 patients from this laboratory ${ }^{7}$ and in a similar survey of $32 \mathrm{PCD}$ patients published recently from the Mayo Clinic. In the Mayo series, 16 antibody-positive patients were identified. ${ }^{17}$ From these reports emerges a rather stereotypic picture of the anti-Yo antibodypositive PCD patient. Always female and usually not previously known to have cancer, she develops over a matter of weeks a severe pancerebellar disorder with truncal and appendicular ataxia, dysarthria, and nystagmus, often with a downbeating component. The clinical findings stabilize within a couple of months, leaving most patients unable to walk without the assistance of one or two people, unable to write legibly, frequently unable to read or watch television because of oscillopsia or diplopia, and having difficulty in being understood when speaking. The behavioral and long-tract signs, when present, are usually mild, and opsoclonus and myoclonus are not characteristic features. Interestingly, two of our patients had progressive prechiasmal visual loss, possibly due to optic neuropathy or retinal degeneration. Most patients remain stable but severely disabled, and except in a few isolated cases, ${ }^{18}$ do not improve with plasmapheresis or immunosuppression. With few exceptions, the anti-Yo antibody-positive PCD patient is eventually found to have an associated gynecologic cancer.

Several different autoantibodies have been identified in patients with PCD (table 2). In comparison with anti-Yo-positive PCD patients, those without the anti-Yo antibody, though pathologically similar, are frequently clinically distinct, and make up a heterogeneous group. Taken together, patients without the anti-Yo antibody have an approximately equal sex distribution, a variety of associated malignancies (most commonly bronchial carcinoma and Hodgkin's lymphoma), and more frequently present with their neurologic syndrome after the malignancy has been diagnosed. Particular subsets of patients with PCD are discussed in the following two papers and elsewhere. ${ }^{3-7,17,19,20}$

With the data reported here and with other recently reported cases or series of PCD, it seems likely that PCD is a group of disorders each related to a particular cancer or cancers. The utility of differentiating between the different anti-Purkinje cell antibodies is that when a particular antibody is present, such as anti-Yo, it strongly suggests the presence of an underlying neoplasm and directs the search for the occult malignancy to one or a few organs. We believe that the presence of the anti-Yo antibody warrants an aggressive diagnostic approach that begins with careful breast and pelvic examination, mammography, pelvic CT, and measurement of ovarian tumor antigen CA 125 . If no malignancy is revealed with this initial workup, repeat mammography, pelvic examination under anesthesia, and uterine D \& C should be performed. If there is still no cancer evident, surgical exploration and removal of pelvic organs may be warranted, particularly in the postmenopausal woman.

Unfortunately, early detection and even cure of the underlying cancer does not usually affect the neurologic symptoms. Although there are occasional reports of patients with paraneoplastic syndromes responding to treatment of the underlying tumor, ${ }^{21}$ none of the patients in this series substantially improved after successful treatment of the cancer and follow-up of more than 4 years. In none of the patients has there been progression of the cerebellar dysfunction after treatment of the cancer; however, in most of the patients, the cerebellar dysfunction had stabilized by the time the cancer diagnosis was made and treatment instituted. Interestingly, the anti-Yo antibody has persisted in all the patients we have followed after what is assumed to be successful treatment of the cancer. In two patients in remission for more than 4 years, the antibody persists in the serum although there has been no progression of their cerebellar dysfunction or evidence of recurrent tumor. Thus, the major importance of identification of the anti-Yo antibody is that early diagnosis leads to treatment, and at times cure, of the cancer. Identification of the antibody does not appear to be helpful in ameliorating the neurologic disease.

The pathogenesis of anti-Yo-positive PCD is unknown. The high titer of antibody suggests an autoimmune mechanism, but there are no reports of successful attempts to reproduce the disease in experimental animals. A recent report of intrathecal injection of anti-Yo antibodies into experimental animals was apparently successful in getting the antibody to the Purkinje cells but not in causing neurologic dysfunction. ${ }^{33}$ Furthermore, autopsy studies indicate that in most patients there is no inflammation around the degenerated Purkinje cells. Thus, there is no direct evidence for either a humoral- or cell-mediated immune reaction in patients with anti-Yo-positive PCD. Further work will be necessary to establish the pathogenesis of the disorder.

\section{References}

1. Brain WR, Daniel PM, Greenfield JG. Subacute cortical cerebellar degeneration and its relation to carcinoma. $J$ Neurol Neurosurg Psychiatry 1951;14:59-75.

2. Brain WR, Wilkinson M. Subacute cerebellar degeneration associated with neoplasms. Brain 1965;88:465-478.

3. Henson RA, Urich $H$, eds. Cancer and the nervous system: the neurological manifestations of systemic disease. Oxford, UK: Blackwell Scientific, 1982:346-367.

4. Hammack JE, Posner JB. Paraneoplastic cerebellar degeneration. In: Plaitakis A, ed. Cerebellar degenerations: clinical neurobiology. Boston: Kluwer Academic, 1992.

5. Anderson NE, Rosenblum MK, Graus F, Wiley RG, Posner JB. Autoantibodies in paraneoplastic syndromes associated with small-cell lung cancer. Neurology 1988;38:1391-1398.

6. Dalmau J, Graus F, Rosenblum MK, Posner JB. Anti-Hu associated paraneoplastic encephalomyelitis/sensory neuronopathy: a clinical study of 71 patients. Medicine 1992;71:59-72. 
7. Anderson NE, Rosenblum MK, Posner JB. Paraneoplastic cerebellar degeneration: clinical-immunological correlations. Ann Neurol 1988;24:559-567.

8. Jaeckle KA, Graus F, Houghton A, Cardon-Cardo C, Nielsen SL, Posner JB. Autoimmune response of patients with paraneoplastic cerebellar degeneration to a Purkinje cell cytoplasmic protein antigen. Ann Neurol 1985;18:592-600.

9. Cunningham J, Graus F, Anderson N, Posner JB. Partial characterization of the Purkinje cell antigens in paraneoplastic cerebellar degeneration. Neurology 1986;36:11631168 .

10. Dropcho EJ, Chen Y, Posner JB, Old LJ. Cloning of a brain protein identified by autoantibodies from a patient with paraneoplastic cerebellar degeneration. Proc Natl Acad Sci USA 1987;84:4552-4556.

11. Furneaux HM, Dropcho EJ, Barbut D, et al. Characterization of a cDNA encoding a $34-\mathrm{kDa}$ Purkinje neuron protein recognized by sera from patients with paraneoplastic cerebellar degeneration. Proc Natl Acad Sci USA 1989;86:2873-2877.

12. Furneaux HM, Rosenblum MK, Dalmau J, Wong E, Woodruff P, Posner JB. Selective expression of Purkinje cell antigens in tumor tissue derived from patients with paraneoplastic cerebellar degeneration. $N$ Engl J Med 1990;322:1844-1851.

13. Chen Y, Rettig WJ, Yenamandra AK, et al. Cerebellar degeneration-related (CDR) antigen: a highly conserved neuroectodermal marker mapped to chromosome $\mathrm{X}$ in human and mouse. Proc Natl Acad Sci USA 1990;87:3077-3081.

14. Fathallah-Shaykh H, Wolf S, Wong E, Posner JB, Furneaux HM. Cloning of a leucine zipper protein recognized by the sera of patients with antibody-associated paraneoplastic cerebellar degeneration. Proc Natl Acad Sci USA 1991;88:3451-3454.

15. Anderson NE, Posner JB, Sidtis JJ, et al. The metabolic anatomy of paraneoplastic cerebellar degeneration. Ann Neurol 1988;23:533-540.

16. Graus F, Abos J, Roquer J, Mazzara R, Pereira A. Effect of plasmapheresis on sexum and CSF autoantibody levels in CNS paraneoplastic syndromes. Neurology 1990;40:16211623.

17. Hammack JE, Kimmel DW, O'Neill BP, Lennon VA. Paraneoplastic cerebellar degeneration: a clinical comparison of patients with and without Purkinje cell cytoplasmic antibodies. Mayo Clin Proc 1990;65:1423-1431.

18. Cocconi G, Ceci G, Jinvarra G, et al. Successful treatment of subacute cerebellar degeneration in ovarian carcinoma with plasmapheresis: a case report. Cancer 1985;56:2318-2320.

19. Stefansson K, Antel JP, Wollman RL, Levin KH, Larson R, Arnason BGW. Anti-neuronal antibodies in serum of a patient with Hodgkin's disease and cerebellar ataxia [abstract]. Neurology 1981;31(No. 4, Pt 2):126.
20. Trotter JL, Hendin BA, Osterland CK. Cerebellar degeneration with Hodgkin's disease: an immunological study. Arch Neurol 1976;33:660-661.

21. Kearsley JH, Johnson P, Halmagyi GM. Paraneoplastic cerebellar disease; remission with excision of the primary tumor. Arch Neurol 1985;42:1208-1210.

22. Brown RH Jr, Ronthal M, Come S, et al. Antibodies to 85,000 dalton protein in paracarcinomatous cerebellar degeneration [abstract]. Neurology 1985;35(suppl 1):288.

23. Kornguth S, Kalinke T, Grunwald G, Brooks B, Zimmerman E. Anti-neurofilament antibodies associated with paraneoplastic syndromes [abstract]. Fed Proc 1985;44:966.

24. Rodriguez M, Truh LI, O'Neill BP, Lennon VA. Autoimmune paraneoplastic cerebellar degeneration: ultrastructural localization of antibody-binding sites in Purkinje cells. Neurology 1988;38:1380-1386.

25. Tanaka K, Yamazaki M, Sato S, Toyoshima I, Yamamoto A, Miyatake T. Antibodies to brain proteins in paraneoplastic cerebellar degeneration. Neurology 1986;36:1169-1172.

26. Greenlee JE, Brashear HR. Antibodies to cerebellar Purkinje cells in patients with paraneoplastic cerebellar degeneration and ovarian carcinoma. Ann Neurol 1983;14:609-613.

27. Greenlee JE, Brashear HR, Jaeckle KA, Stroop WG. Anticerebellar antibodies in sera of patients with paraneoplastic cerebellar degeneration: studies of antibody specificity and response to plasmapheresis [abstract]. Ann Neurol $1986 ; 20: 139$.

28. Greenlee JE, Lipton HL. Anticerebellar antibodies in serum and cerebrospinal fluid of a patient with oat cell carcinoma of the lung and paraneoplastic cerebellar degeneration. Ann Neurol 1986;19:82-85.

29. Bourdette DN, Nilaver G. Cerebellar degeneration associated with anti-Purkinje cell antibodies and benign IgG monoclonal gammopathies [abstract]. Neurology 1987;37(suppl 1): 291 .

30. Royal W III, Galasko DR, McKhann GM, Cunningham JM, Dropcho EJ. Clinical course, immunologic, and biochemical features of a patient with paraneoplastic cerebellar dysfunction [abstract]. Neurology 1987;37(suppl 1):305-306.

31. Anderson NE, Budde-Steffen C, Wiley RG, et al. A variant of the anti-Purkinje cell antibody in a patient with paraneoplastic cerebellar degeneration. Neurology 1988;38:10181026.

32. Tsukamoto T, Yamamoto H, Iwasaki Y, Yoshie O, Terunuma H, Suzuki H. Antineural autoantibodies in patients with paraneoplastic cerebellar degeneration. Arch Neurol 1989;46:1225-1229.

33. Graus F, Illa I, Agusti M, Ribalta T, Cruz-Sanchez F, Juarez C. Effect of intraventricular injection of an anti-Purkinje cell antibody (anti-Yo) in a guinea pig model. J Neurol Sci $1991 ; 106: 82-87$. 


\section{Neurology}

\section{Paraneoplastic cerebellar degeneration.: I.A clinical analysis of 55 anti-Yo antibody -positive patients}

K. Peterson MD, M. K. Rosenblum, H. Kotanides MS, et al. Neurology 1992;42;1931

DOI 10.1212/WNL.42.10.1931

\section{This information is current as of October 1,1992}

\section{Updated Information \&}

Services

Citations

Permissions \& Licensing

Reprints including high resolution figures, can be found at: http://n.neurology.org/content/42/10/1931.full

This article has been cited by 22 HighWire-hosted articles: http://n.neurology.org/content/42/10/1931.full\#\#otherarticles

Information about reproducing this article in parts (figures,tables) or in its entirety can be found online at:

http://www.neurology.org/about/about_the_journal\#permissions

Information about ordering reprints can be found online:

http://n.neurology.org/subscribers/advertise

Neurology ${ }^{\circledR}$ is the official journal of the American Academy of Neurology. Published continuously since 1951, it is now a weekly with 48 issues per year. Copyright $(9) 1992$ by Edgell Communications, Inc.. All rights reserved. Print ISSN: 0028-3878. Online ISSN: 1526-632X.

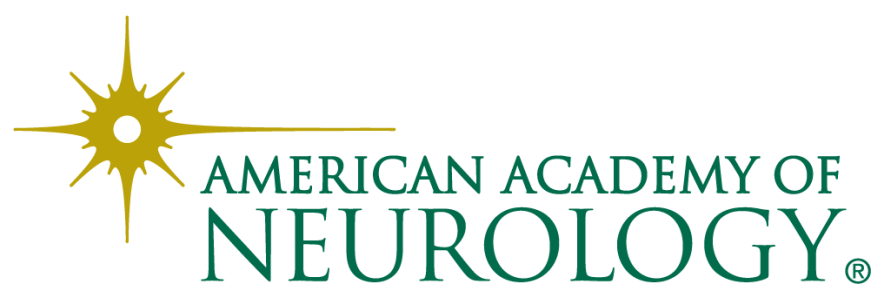

